\title{
Arbitrary Lagrangian-Eulerian method for non-linear problems of geomechanics
}

\author{
M Nazem ${ }^{1,3}$, J P Carter ${ }^{1}$ and D W Airey ${ }^{2}$ \\ ${ }^{1}$ Centre for Geotechnical and Materials Modelling, The University of Newcastle, \\ Callaghan NSW 2308, Australia \\ ${ }^{2}$ Centre for Geotechnical Research, The University of Sydney \\ Sydney NSW 2308 Australia
}

\begin{abstract}
In many geotechnical problems it is vital to consider the geometrical non-linearity caused by large deformation in order to capture a more realistic model of the true behaviour. The solutions so obtained should then be more accurate and reliable, which should ultimately lead to cheaper and safer design. The Arbitrary Lagrangian-Eulerian (ALE) method originated from fluid mechanics, but has now been well established for solving large deformation problems in geomechanics. This paper provides an overview of the ALE method and its challenges in tackling problems involving non-linearities due to material behaviour, large deformation, changing boundary conditions and time-dependency, including material rate effects and inertia effects in dynamic loading applications. Important aspects of ALE implementation into a finite element framework will also be discussed. This method is then employed to solve some interesting and challenging geotechnical problems such as the dynamic bearing capacity of footings on soft soils, consolidation of a soil layer under a footing, and the modelling of dynamic penetration of objects into soil layers.
\end{abstract}

\section{Introduction}

The finite element method plays an important role in solving non-linear problems of geomechanics in cases where an analytical solution cannot be obtained due to complexity, which often arises because of the non-linear nature of the problem. Non-linear material behaviour, large deformations, changing boundary conditions, and time dependency of behaviour are the main sources of non-linearity in geotechnical problems. Conventional finite element methods, such as the Updated-Lagrangian method, often fail in the analysis of such non-linear problems, mainly due to mesh distortion. In order to tackle these problems successfully, two main strategies, viz., h-adaptivity and r-adaptivity, have been introduced into the finite element approach.

The h-adaptive finite element method can overcome mesh distortion by generating a new mesh based on further sub-division of the area of basic elements where the interpolation should be improved or where mesh distortion occurs. Zienkiewicz and Huang [1] presented a successful application of the h-adaptive finite element method which they used to analyse the localisation occurring in geomechanics problems such as footings and slopes. Hu and Randolph [2] also suggested a numerical procedure which combined the conventional small strain finite element method with an automatic

\footnotetext{
${ }^{3}$ Correspondence to: Majidreza.Nazem@newcastle.edu.au; Fax: +61 249216991.
} 
mesh generation algorithm and a linear stress interpolation technique to deal with mesh distortion. They used this method to study plane strain and axi-symmetric problems such as cavity expansion and the bearing capacity of footings. They found this technique to be a practical approach which makes good use of well established small strain finite element code and yet provides accurate solutions for problems that involve large strains and deformations.

The r-adaptive finite element method, on the other hand, attempts to eliminate the mesh distortion by relocating the nodes in the finite element domain. Unlike the h-adaptive technique, the radaptive strategy retains the advantage of not changing the number of nodes or the connectivity of the elements. The Arbitrary Lagrangian-Eulerian (ALE) method is probably the most well-known radaptive technique, and it is based on the concept of separating the mesh displacements from the material displacements. The Arbitrary Lagrangian-Eulerian method has been well developed for many geotechnical applications such as general large deformation problems [3], consolidation problems [4], dynamic analysis [5] and the dynamic penetration of objects into soil [6]. This paper summarises some of the recent developments of the ALE method, specifically in geotechnical engineering.

\section{ALE method}

The Lagrangian finite element methods, such as the Updated-Lagrangian (UL) method, normally fail to analyse accurately those problems involving relatively large deformations which lead to severe distortion of the finite element mesh used to represent the continuum. In these Lagrangian methods, a computational grid is connected to the material particles, following them as deformation occurs in the finite element domain. Such a permanent connection can potentially lead to mesh distortion, ultimately identified by a negative Jacobian of an individual element or elements, or a significant loss of precision in the finite element solution. The Arbitrary Lagrangian-Eulerian (ALE) method, on the other hand, eliminates the mesh distortion by separating the material displacements from the grid (or mesh) displacements. The equilibrium equation in the ALE method can be written in either a coupled form or a decoupled form. In its coupled form, the global equations contain two set of unknowns that include the material displacements and the grid (or mesh) displacements. The coupled ALE procedure is normally slower than the uncoupled method since the number of unknowns is doubled and the convection of the state variables, i.e., the remapping of all variables from the old mesh to the new mesh, is normally carried out during each iteration. Nonetheless, this method guarantees that the state variables will satisfy the global equilibrium as well as any local plasticity consistency relations at the end of each iteration. Compared to the coupled form, the decoupled form of the ALE method, also termed the 'operator-split technique', has the advantage of significantly reducing computation times since the global equations in this method are written in terms of material displacements only. Analysis by the operator-split technique usually includes two main steps: an ordinary Updated-Lagrangian (UL) step, aimed at finding the material displacements, followed by an Eulerian step, which eliminates the mesh distortion and convects the state variables. The operator-split technique is necessarily more efficient than the coupled ALE procedure, since the number of unknowns in this method is only half of the unknowns in the coupled ALE. In addition, mesh refinement and transformation of state parameters are conducted only once at the end of each time step. The ALE method used in this study is based upon the operator-split technique described in [3]. A summary of the method and its key aspects in different geotechnical applications are presented in the following sections of this paper.

\subsection{Solid mechanics}

Soon after the introduction of the ALE method in fluid mechanics, various researchers extended its application to problems of solid mechanics. Benson [7] introduced the operator split technique to separate the computational material domain from the grid domain. Benson showed that this technique can reduce the analysis time by a factor of about two. This technique has been widely used for analysing non-linear problems of solid mechanics, such as metal powder compaction, forming, cutting and guillotining. Nazem et al. [3] described one of the earliest applications of the operator split 
technique in geomechanics. In the UL step of the operator-split technique, the material displacements are calculated using the principle of virtual displacements, as follows

$$
\int_{V} \sigma_{i j} \delta \varepsilon_{i j} d V=\int_{V} \delta u_{i} b_{i} d V+\int_{S} \delta u_{i} q_{i} d S
$$

where $\sigma$ is the Cauchy stress tensor, $\delta \varepsilon$ denotes the variation of strain due to virtual displacement $\delta u, u$ represents the material displacements, $b$ is the body force, $q$ is the surface load acting on area $S$ of volume $V$. The Eulerian step includes mesh refinement followed by the transformation of state variables. For regular domains a structured mesh generation algorithm may be used to refine the finite element mesh, without changing the topology [8]. Alternatively, a refined mesh can be obtained by performing a static analysis [3]. After mesh refinement, the state variables, such as the stresses and hardening parameters, need to be remapped from the material domain into the new grid domain. The remapping is usually conducted using the convection equation, obtained from a first order expansion of Taylor's series [9], i.e.,

$$
d f^{r}=d f+\left(u_{i}-u_{i}^{r}\right) \cdot \frac{\partial f}{\partial x_{i}}
$$

where $f^{r}$ represents the value of an arbitrary state variable $f$ in the mesh domain and $u^{r}$ denotes the mesh displacements. Note that the state variables transformed by equation (2) may not satisfy the global equilibrium and the principle of plasticity consistency, demanding more equilibrium iterations. Alternatively, the unbalanced force due to the remapping can be calculated and carried over to the next load increment.

\subsection{Coupled analysis}

In some geotechnical problems, such as the consolidation of soils, deformations are coupled with porewater pressures. The global equations for such problems may be obtained by combining the principle of virtual displacements and the continuity equation through the principle of effective stress and Darcy's law [4]. The matrix form of the coupled global finite element equations may be written as

$$
\left[\begin{array}{cc}
\mathbf{K} & \mathbf{L} \\
\mathbf{L}^{\mathrm{T}} & \mathbf{0}
\end{array}\right]\left\{\begin{array}{c}
\dot{\mathbf{U}} \\
\dot{\mathbf{P}}
\end{array}\right\}+\left[\begin{array}{cc}
\mathbf{0} & \mathbf{0} \\
\mathbf{0} & \dot{\mathbf{H}}
\end{array}\right]\left\{\begin{array}{l}
\mathbf{U} \\
\mathbf{P}
\end{array}\right\}=\left\{\begin{array}{l}
\dot{\mathbf{F}}^{\mathrm{ext}} \\
\dot{\mathbf{Q}}^{\mathrm{ext}}
\end{array}\right\}
$$

where $\mathbf{K}, \mathbf{L}$ and $\mathbf{H}$ are the global stiffness, coupling and flow matrices respectively, $\mathbf{U}$ denotes the nodal displacements vector, $\mathbf{P}$ is the nodal pore-water pressure vector, $\mathbf{F}^{\text {ext }}$ and $\mathbf{Q}^{\text {ext }}$ represent the external force vector and the fluid supply vector, respectively. Details of these matrices and vectors can be found in [4]. To obtain the values of displacements and pore-water pressures in each UL step, equation (3) needs to be integrated over the time. Note that in an Eulerian step of a coupled analysis, the nodal values of pore-water pressure as well as other state variables at Gauss points need to be transformed from the old material domain into the new grid configuration.

An important issue which arises in a large deformation coupled analysis is the change in permeability (hydraulic conductivity) of the soil. In this study it is assumed that the permeability of the soil depends on its voids ratio which changes as the soil deforms and undergoes volume change according to [4]

$$
e^{t+\Delta t}=J\left(1+e^{t}\right)-1
$$

where $e$ is the voids ratio and $J$ denotes the Jacobian of the deformation gradient tensor. In addition, in a large deformation analysis the permeability of the soil may also change if the soil is initially anisotropic and elements subsequently undergo significant rigid body rotation. This effect is expressed by [13] 


$$
k_{i j}^{t+\Delta t}=R_{i k}^{\mathrm{T}} \cdot k_{k l}^{t} \cdot R_{l j}
$$

where $k_{i j}$ represents the permeability tensor and $R_{i j}$ is the rotation matrix at a Gauss point, which corresponds to the rotation of a vector in a Cartesian system of coordinates by a defined angle about the origin.

\subsection{Dynamic and contact analysis}

The effect of inertia forces should not be neglected in problems involving relatively fast loading rates. The rapid penetration of objects into a layer of soil is a classical example of such problems. This type of problem contains another complexity due to the boundary conditions changing continuously during the analysis. The principle of virtual work for the dynamic analysis of a system of $k$ bodies in contact can be written as

$$
\begin{aligned}
& \sum_{k}\left(-\int_{V_{k}} \sigma_{i j} \delta \varepsilon_{i j} d V_{k}-\int_{V_{k}} \delta u_{i} \rho \ddot{u}_{i} d V_{k}-\int_{V_{k}} \delta u_{i} c \dot{u}_{i} d V_{k}\right)+\sum_{k}\left(\int_{V_{k}} \delta u_{i} b_{i} d V_{k}+\int_{S_{k}} \delta u_{i} q_{i} d S_{k}\right) \\
& +\int_{S_{c}}\left(t_{N} \delta g_{N}+t_{T} \delta g_{T}\right) d S_{c}=0
\end{aligned}
$$

where $\dot{u}$ and $\ddot{u}$ represent material velocities and accelerations, respectively, $\rho$ and $c$ are the material density and damping, $\delta g_{N}$ and $\delta g_{T}$ denote the virtual normal and tangential gap displacements (between the bodies 'in contact'), $t_{N}$ and $t_{T}$ are the normal and tangential forces at the contact surface $S_{c}$. In order to describe the contact at the interface between two bodies, constitutive equations must be provided for the tangential and normal directions. Among several strategies available in contact mechanics we use the penalty method to formulate these constitutive relations. For more details see [10] and [11].

The analysis of dynamic problems by the ALE method requires further considerations. First, the velocities and accelerations of all nodal points must be remapped from the material configuration to the grid computation in the Eulerian step. Second, the computational boundaries must be able to absorb the energy of oncoming waves. In other words, the outgoing waves from a source must not reflect back from truncated artificial boundaries defined in a finite element domain. This can be assured by using a standard viscous boundary [5]. Last, but not least, it is noted that the soil constitutive relations may be affected by the rate of loading. For example, the undrained shear strength of cohesive soils, $s_{u}$, often increases with the rate of straining [12]. In the examples discussed below this effect has been described by

$$
s_{u}=s_{u, r e f}\left[1+\lambda \log \left(\frac{\dot{\gamma}}{\dot{\gamma}_{\text {ref }}}\right)\right]
$$

where $s_{u, r e f}$ is a reference undrained shear strength measured at a reference shear strain rate of $\dot{\gamma}_{\text {ref }}, \lambda$ denotes the rate of increase per log cycle of time and $\dot{\gamma}$ denotes the shear strain rate.

\section{Numerical examples}

Experimental as well as numerical validation of the ALE method reviewed here has been reported previously $[3,4,5,6]$. To demonstrate its abilities and its wide range of application, the ALE method has been used to analyse some interesting geomechanics problems, including the static and dynamic analysis of a footing resting on an undrained soil layer, the consolidation analysis of soil under a rigid footing and the deep penetration of a free-falling penetrometer into an undrained soil layer. These problems have been solved numerically using SNAC, a finite element code developed by the geotechnical research group at the University of Newcastle, Australia. 


\subsection{Static and dynamic analysis of a rigid footing on an undrained soil layer}

In the first example, we consider a rigid strip footing on an undrained soil layer subjected to static as well as dynamic loads. Due to symmetry, only the right-hand half of the footing is analysed using the finite element mesh and the boundary conditions shown in Figure 1. The mesh consists of 3884 6noded plane-strain triangular elements, with 7903 nodal points overall. In the dynamic analyses, the bottom and right boundaries are modelled as energy absorbing boundaries in order to avoid reflection of oncoming waves. Table 1 denotes the material properties used in all analyses performed in this example. An associated Tresca model was used to represent a purely cohesive soil deforming under undrained conditions. Note that the smallest element dimension under the footing is equal to $B / 16$,

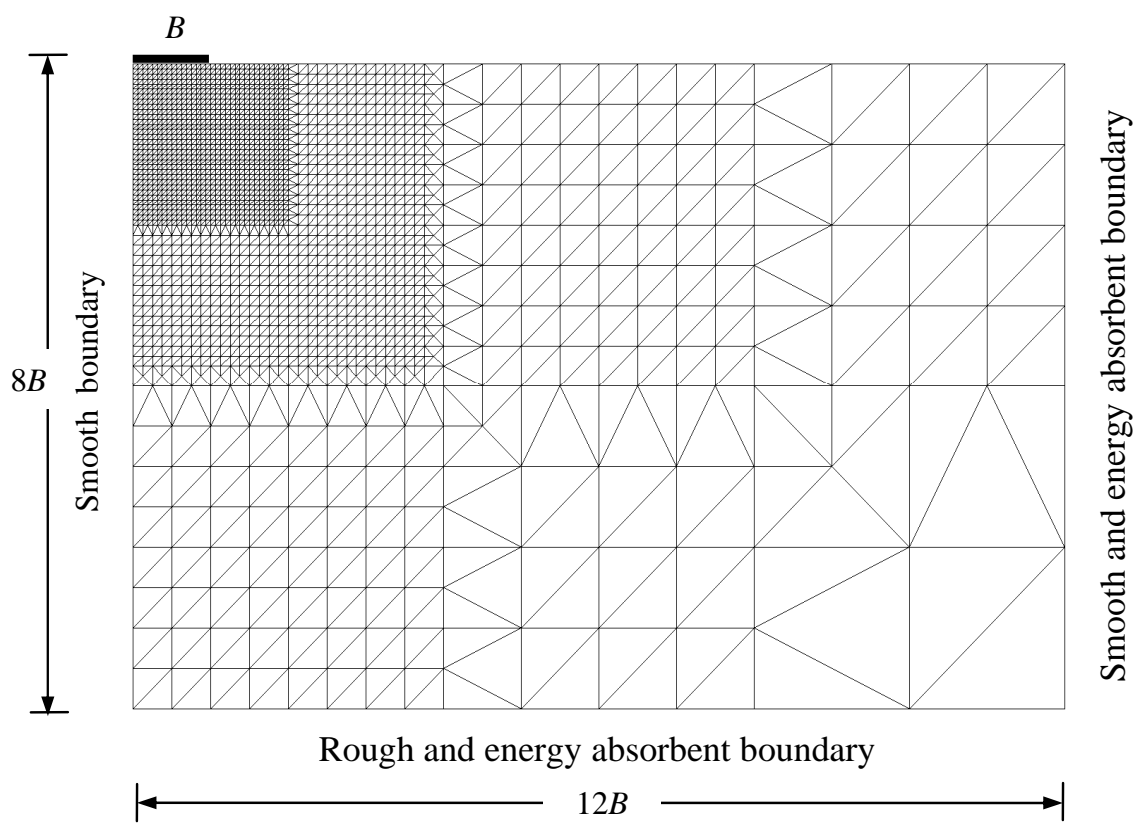

Figure 1. Rigid strip footing; geometry, finite element mesh and boundary conditions.

where $B$ is the footing half-width, making the problem vulnerable to mesh distortion but potentially

Table 1. Material properties used in Examples 3.1 and 3.3.

\begin{tabular}{|l|c|l|}
\hline \multicolumn{1}{|c|}{ Material Property } & Value & \multicolumn{1}{c|}{ Comments } \\
\cline { 1 - 1 } Undrained Young's modulus, $E_{u}$ & \multirow{2}{*}{$E_{u} / s_{u, \text { ref }}=100$} & To represent a relatively soft soil \\
\hline Undrained shear strength, $s_{u, r e f}$ & 0.49 & $\begin{array}{l}\text { To represent the elastic incompressibility } \\
\text { of soil under undrained conditions }\end{array}$ \\
\hline Undrained Poisson's ratio, $v_{u}$ & 2 tonne $/ \mathrm{m}^{3}$ & Corresponds to unit weight of $19.6 \mathrm{kN} / \mathrm{m}^{3}$ \\
\hline Unit mass, $\rho$ &
\end{tabular}

increasing the accuracy of the analysis. As reported earlier, the UL method is not normally able to provide a solution to such problems due to significant mesh distortion occurring in the areas under and next to the footing where intense shearing is likely to occur [3,4]. For this reason, only the ALE results are reported in this study.

For the dynamic analysis a prescribed vertical velocity, $0.5 \mathrm{~m} / \mathrm{sec}$, was applied to the footing during a time interval of $1 \mathrm{sec}$ using 500 equal time steps. One static and three dynamic analyses, 


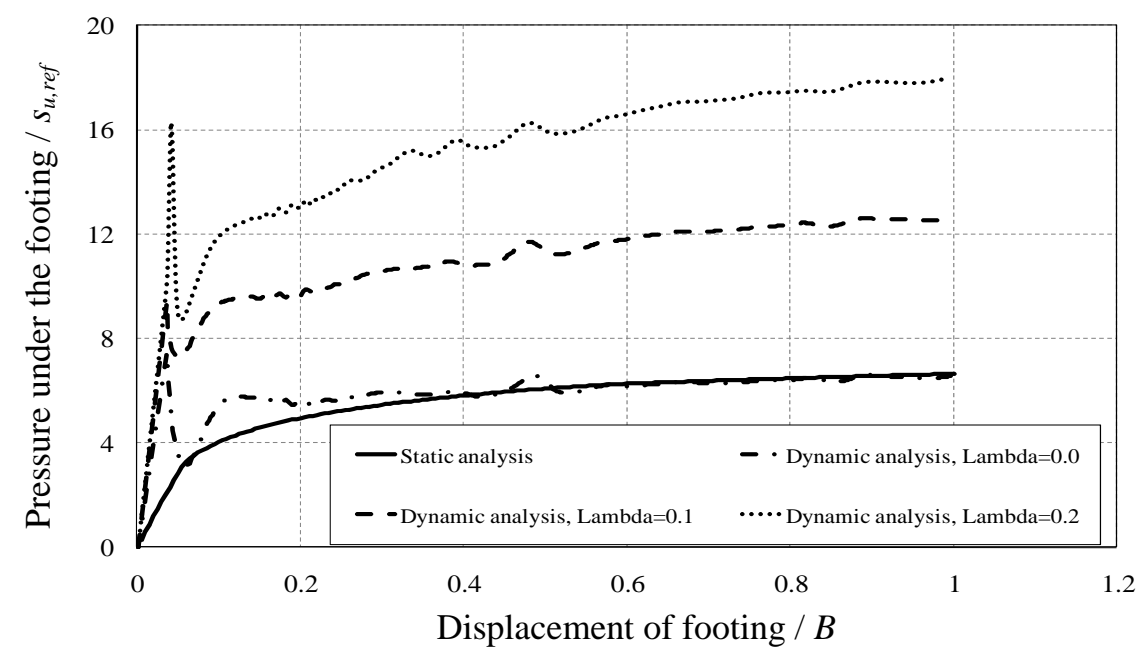

Figure 2. Average pressure on the footing versus prescribed displacement.

assuming $\lambda=0.0,0.1$, and 0.2 (see Equation (7)), were performed. The average pressure predicted under the footing, normalised by $s_{u \text { uref }}$, is plotted versus its vertical displacement, normalised by $B$, in Figure 2. It is worth noting that an increase in the rate parameter $\lambda$ significantly increases the mobilised strength of the soil under dynamic loading and the inertia effects abate relatively quickly after the initial impact.

\subsection{Consolidation of an elastoplastic soil layer under a rigid footing}

In the second example, the consolidation of a soil layer under a rigid impermeable footing, assuming small deformations as well as large deformations, is considered. The geometry and boundary

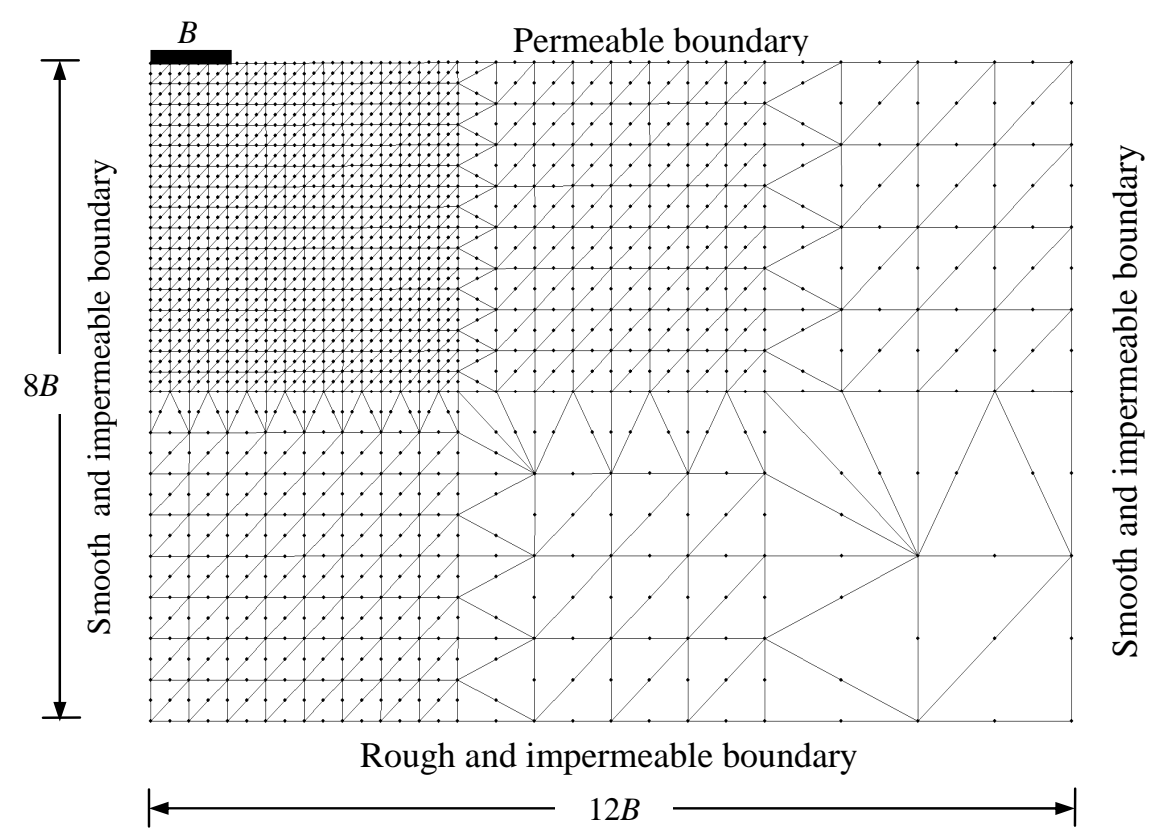

Figure 3. Rigid strip footing; geometry, finite element mesh and boundary conditions. 
conditions are shown in Figure 3. Note that in a coupled displacement-pore water pressure analysis it is actually the drained soil properties that are required. The drained Young's modulus, $E^{\prime}$, and the drained cohesion, $c^{\prime}$, of the soil are assumed to be $500 \mathrm{kPa}$ and $5 \mathrm{kPa}$, respectively. The drained friction angle, $\varphi^{\prime}$, is $20^{\circ}$ whilst the dilation angle was assumed to be zero. The initial (isotropic) permeability of the soil in the $x$ and $y$ directions is $10^{-4} \mathrm{~m} /$ day and the unit weight of the pore water and the soil are $10 \mathrm{kN} / \mathrm{m}^{3}$ and $20 \mathrm{kN} / \mathrm{m}^{3}$, respectively. Poisson's ratio of the soil skeleton was assumed to be 0.3. The water table is located at the ground surface. Assuming $B=0.5 \mathrm{~m}$ and small strains, the theoretical undrained bearing capacity of the soil under the footing is approximately $102 \mathrm{kPa}$.

The analysis was performed in three stages. In the first stage, body force due to self-weight of the soil was applied very slowly to produce fully drained conditions and an initial geostatic stress field, i.e., zero excess pore pressure. In the second stage, a uniform pressure of $q$ was applied to the footing at a uniform rate over a period of 1 day, i.e., relatively rapid loading. Two different values of $q=90 \mathrm{kPa}$ and $q=150 \mathrm{kPa}$ were used for this step. The soil was then permitted to consolidate over time, keeping the load constant. The settlement of the footing, normalised by $B$, versus time is plotted in Figure 4 for the second and the third stage of the analysis. For $q=90 \mathrm{kPa}$, the final consolidation settlement of the footing predicted by small deformation analysis and the ALE analysis are $0.86 \mathrm{~B}$ and $0.75 B$, respectively, occurring after approximately 3100 and 3500 days. Compared with the small deformation analysis, the final consolidation settlement predicted by the ALE method occurs over a relatively longer period of time, mainly due to the changes in the permeability during consolidation. Note that as the soil is deformed and consolidated, its permeability changes with its void ratio (equation 5) as well as potentially with rigid body rotation (equation 6). For $q=150 \mathrm{kPa}$, the final consolidation settlement predicted by the ALE method is $1.41 B$. Note that small deformation analysis cannot predict a solution in this case since the pressure applied rapidly on the footing exceeds the (small strain) undrained bearing capacity of the soil.

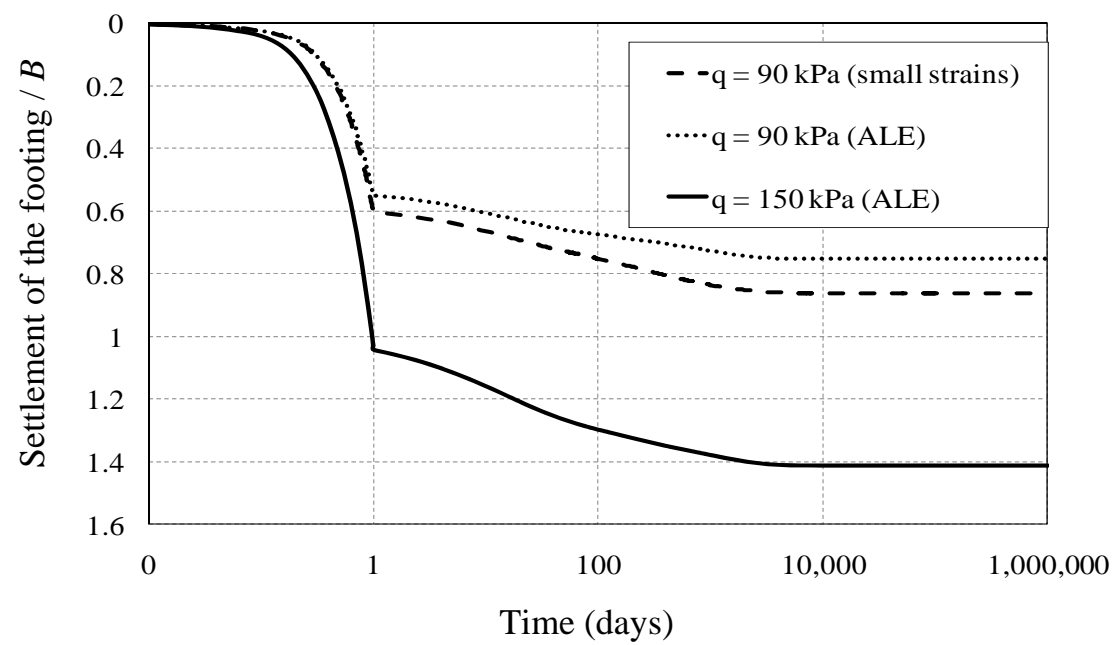

Figure 4. Normalised settlement of the footing versus time.

\subsection{Analysis of a free-falling penetrometer}

In this example, the ability of the ALE method in providing an efficient solution to one of the most sophisticated problems in geomechanics, viz., the dynamic penetration of a free-falling penetrometer into an undrained layer of soil, is illustrated. The geometry, finite element mesh and the boundary conditions of the problem are shown in Figure 5. The mass and initial velocity of the penetrometer are assumed to be $0.2 \mathrm{~kg}$ and $10 \mathrm{~m} / \mathrm{sec}$, respectively, i.e., the initial kinetic energy of the object as it first 


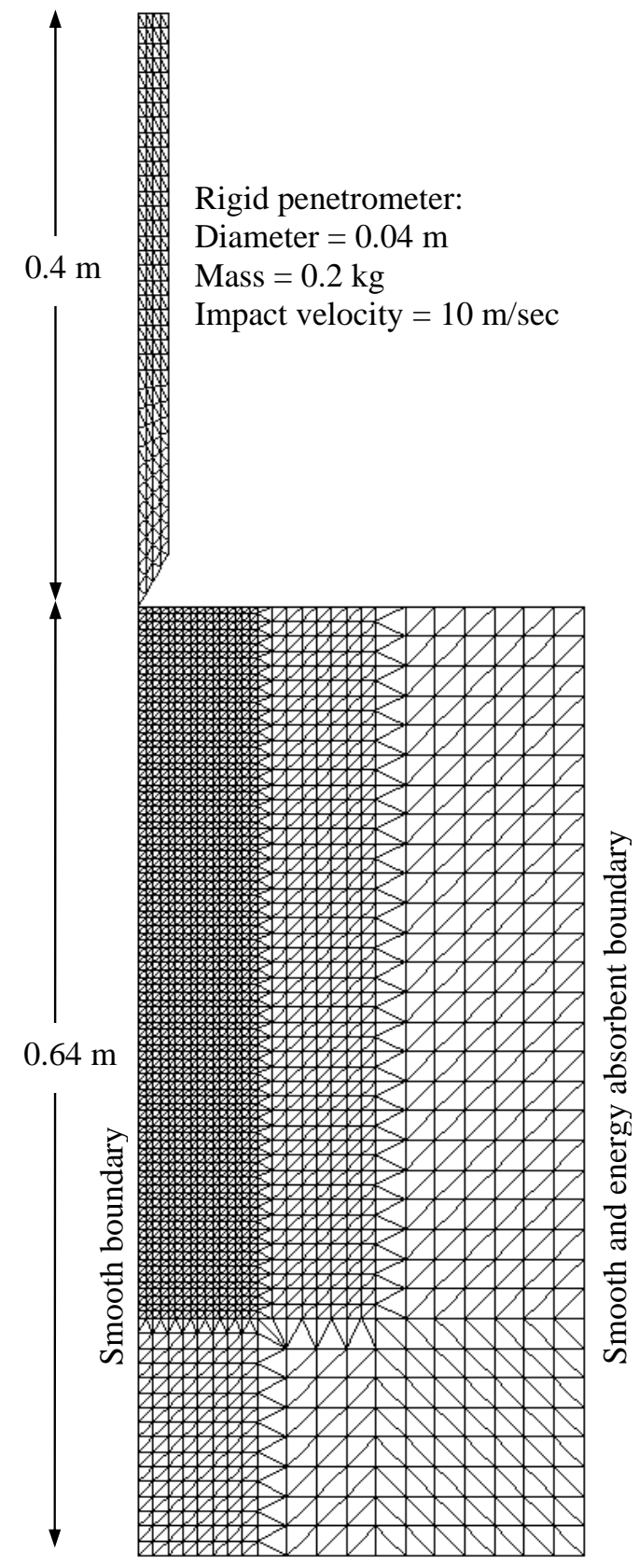

Rough and energy absorbent boundary

$$
0.3 \mathrm{~m}
$$

Figure 5. Finite element mesh of free falling penetrometer.

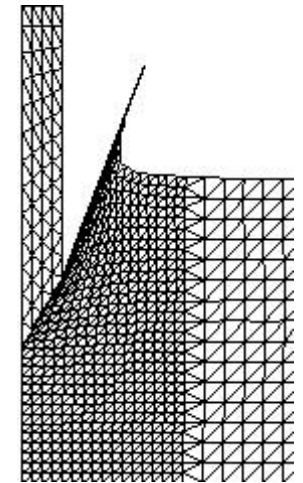

Figure 6. Severe mesh distortion occurring in UL analysis at $t=0.0097 \mathrm{~s}$.

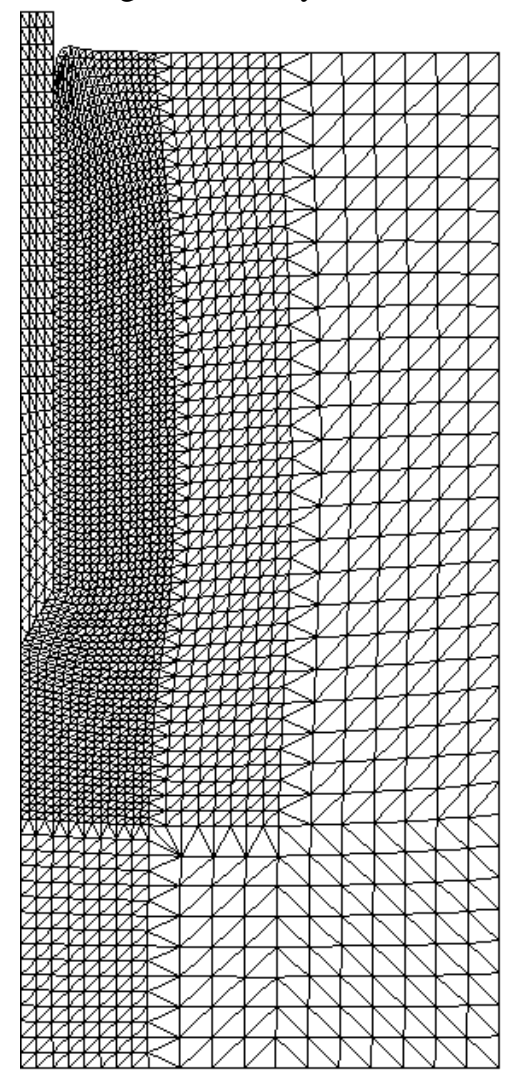

Figure 7. Deformed mesh at the end of ALE analysis.

strikes the soil is 10 Joules. The material properties are the same as the properties used in Example 1 (see Table 1). The strain rate parameter, $\lambda$, is equal to 0.2 . The size of each time step was assumed to be $2 \times 10^{-5}$ sec. 
The analysis by the UL method could not be finalised due to the obvious mesh distortion occurring at increment $485(t=0.0097 \mathrm{sec})$, as depicted in Figure 6. The ALE method was able to perform the analysis successfully and predicted a total penetration of $0.37 \mathrm{~m}$ (9.23 diameters) occurring after 4092 increments (or $0.082 \mathrm{sec}$ ). The final deformed mesh obtained by the ALE method is shown in Figure 7 . The average vertical contact pressure between the penetrometer and the

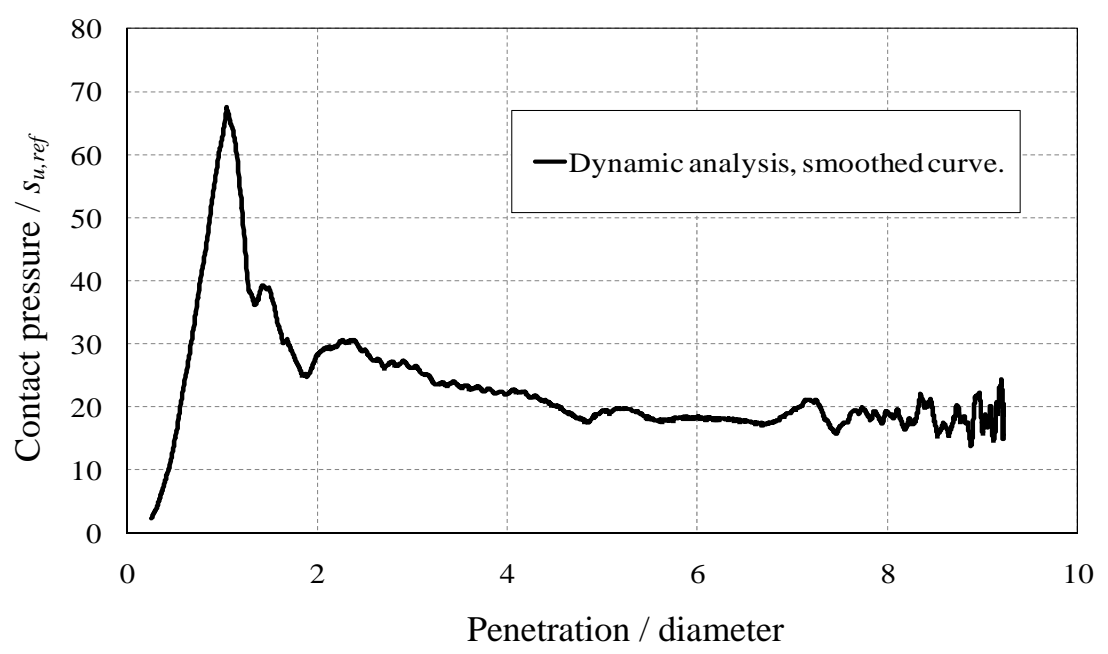

Figure 8. Normalised contact pressure between the penetrometer and soil versus penetration.

soil, normalised by $s_{u, r e f}$, is plotted versus the penetration of the object, normalised by its diameter, in Figure 8. In addition, the vertical velocity of the penetrometer is plotted versus its normalised penetration as in Figure 9, where an approximately parabolic relation can be observed.

\section{Conclusion and future challenges}

The application of the Arbitrary Lagrangian-Eulerian method in solving a wide range of geotechnical

\section{Penetration / diameter}

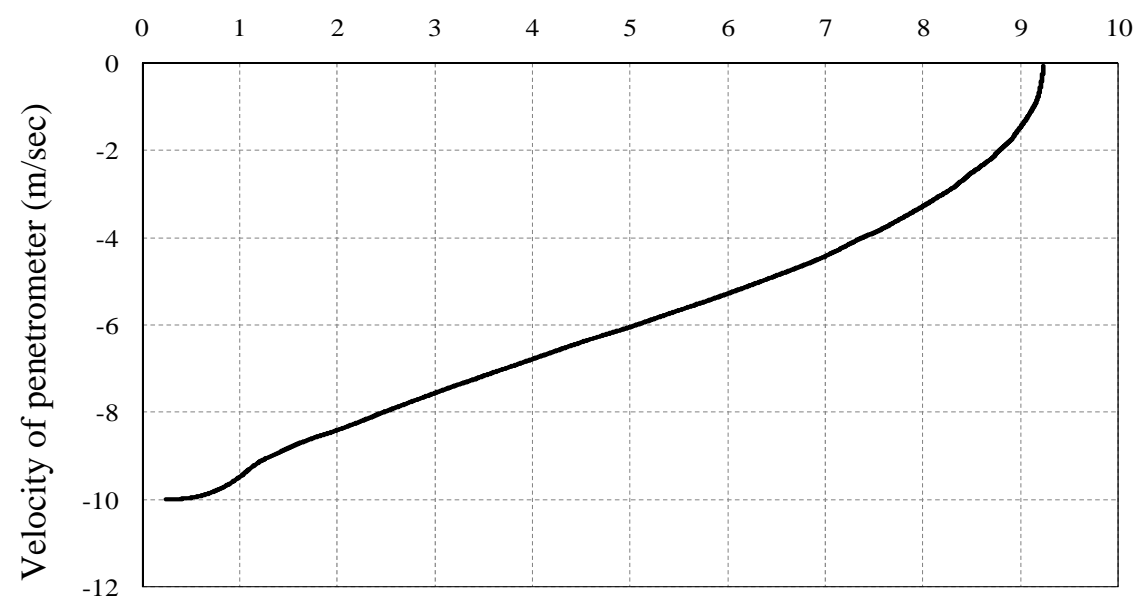

Figure 9.Velocity of the penetrometer versus penetration. 
problems has been presented in this paper. This was achieved by solving some typical examples including the large deformation analysis of a footing under static as well as dynamic loads, the consolidation of a soil layer under a footing and the dynamic penetration of an object into a soil layer. It is concluded that the ALE method can be successfully used in large deformation analysis of geotechnical problems. However, application of the ALE method in the dynamic analysis of coupled displacement-pore water pressure problems in geomechanics has not previously been reported and as such demands further investigation. This will be addressed in future work.

\section{References}

[1] Zienkiewicz OC and Huang M 1995 Localization problems in plasticity using finite elements with adaptive remeshing Int. J. Numer. Anal. Methods Geomech. 89, 127-148.

[2] $\mathrm{Hu}$ Y and Randolph MF 1998 A practical numerical approach for large deformation problems in soils, Int. J. Numer. Anal. Methods Geomech., 22, 327-350.

[3] Nazem M, Sheng D, and Carter JP 2006 Stress integration and mesh refinement in numerical solutions to large deformations in geomechanics Int. J. Numer. Methods. Eng. 65, 1002-1027

[4] Nazem M, Sheng D, Carter JP and Sloan SW 2008 Arbitrary-Lagrangian-Eulerian method for large-deformation consolidation problems Int. J. Numer. Anal. Methods Geomech. 32, 10231050

[5] Nazem M, Carter JP and Airey D 2009 Arbitrary Lagrangian-Eulerian Method for dynamic analysis of Geotechnical Problems Comput. Geotech. 36, 549-557.

[6] Carter JP, Nazem M, Airey DW and Chow SW 2010 Dynamic analysis of free-falling penetrometers in soil deposits. Geotech. Special Pub. 199. Geoflorida 2010 - Advances in Analysis, Modelling and design. American Society of Civil Engineers, West Palm Beach, Florida, 53-68.

[7] Benson DJ 1989 An efficient, accurate and simple ALE method for nonlinear finite element programs Comput. Meth. Appl. Mech. Eng. 72, 305-350

[8] Gadala MS, Movahhedy MR and Wang J 2002 On the mesh motion for ALE modeling of metal forming processes Finite. Elem. Anal. Des. 38, 435-459.

[9] Hughes TJR, Liu WK and Zimmermann TK 1981 Lagrangian-Eulerian finite element formulation for incompressible viscous flow. Comput. Meth. Appl. Mech. Eng 58, 19-36

[10] Sheng D, Wriggers P and Sloan SW 2006 Improved numerical algorithms for friction contact in pile penetration analysis Comput. Geotech. 33, 341-354.

[11] Sheng D, Nazem M and Carter JP 2009 Some computational aspects for solving deep penetration problems in geomechanics Comput. Mech. 44, 549-561.

[12] Graham J, Crooks JHA and Bell AL 1983 Time effects on the stress-strain behaviour of natural soft clays Géotechnique 33, 327-340.

[13] Carter JP, Small JC and Booker JR 1977 A theory of finite elastic consolidation Int. J. Solids Struct. 13, 347-478. 